\title{
ROLE OF ELECTRON CORRELATIONS AND MAGNETISM IN THE STABILITY OF Re DIMERS ON W(110)
}

\author{
A. M. Oleś*, M. C. Desjonquères $\left({ }^{1}\right)$; D. Spanjaard $\left({ }^{2}\right)$ and G. Tréglia $\left({ }^{2}\right)$ \\ ( ${ }^{1}$ IRF/DPhG/PAS-CEN de Saclay, F-91191 Gif-sur-Yvette, France \\ ( $\left.{ }^{2}\right)$ Laboratoire de Physique des Solides, Université Paris-Sud, Centre d'Orsay, F-91405 Orsay, France
}

Abstract. - We study the importance of electron correlations and magnetism in the stability of Re dimers on W(110) surface. It is found that the magnetism of monomers is the principal reason for the instability of $R_{2}$ and that both monomer and dimer are magnetic.

\section{Introduction}

It is rather surprising that two Re atoms do not form a stable dimer on the W(110) surface, as found experimentally $[1,2]$. One expects that the energy should be gained by new bond being formed between two single Re adatoms with a nearly half-filled 5 d shell. Instead, the interaction between them is weak and repulsive at short distances $[2,3]$. Furthermore, the decrease of this interaction energy is found along $5 \mathrm{~d}$ series from $\mathrm{Ta}_{2}$ to $\mathrm{Re}_{2}$, followed by a slight increase from $\mathrm{Re}_{2}$ to $\mathrm{Pt}_{2}$ [2]. Clearly, the situation of $\mathrm{Re}_{2}$ is unique and the key to understand the observed behaviour.

It has been suggested recently that the instability of $\mathrm{Re}_{2}$ may be caused either by electron correlation [4], or by magnetic moments which form on $\mathrm{Re}_{1}$ [5]. Both phenomena should destabilize $\operatorname{Re}_{2}$. So far, they were treated separately: correlation effects within second order perturbation expansion (SOP) and magnetic instability within Hartree-Fock (HF) approximation. It has been found [4] that electron correlations are considerable and one expects that SOP is not sufficient [6]. One cannot also conclude about the nature of the ground state by making HF analysis. Motivated by these facts, we performed a model study of the $\mathrm{Re}_{2}$ instability on W(110) treating local correlations by the method of local ansatz (LA), developed earlier for magnetism of transition metals [7].

2. Model Hamiltonian and calculation method

We study a model Hamiltonian [7]

$$
\begin{aligned}
& H=\sum_{m i \sigma} \varepsilon_{m} n_{m i \sigma}+\sum_{m n i j \sigma} t_{m i, n j} a_{m i \sigma}^{+} a_{n j \sigma}+ \\
& +\frac{1}{2} \sum_{m i j \sigma^{\prime}}\left(U_{m} n_{m i \sigma} n_{m j \sigma^{\prime}}+J_{m} a_{m i \sigma}^{+} a_{m j \sigma^{\prime}}^{+} a_{m i \sigma^{\prime}} a_{m j \sigma}\right),
\end{aligned}
$$

where five equivalent $5 \mathrm{~d}$ orbitals $(i, j)$ at each atom $m(n)$ are assumed. The corresponding local densities of states (LDOS) are found from a continued fraction expansion exact up to the 22nd moment and terminated in the usual way [8]. Thereby, we use the neutrality condition for each atom to determine the site energies $\varepsilon_{m}$ and assume that all $\mathrm{W}$ atoms of the $\mathrm{W}(110)$ surface are nonmagnetic. $U_{m}$ and $J_{m}$ stand for local Coulomb and exchange interaction, respectively. We found that it is sufficient to consider $U_{\mathrm{Re}}=$ $U_{\mathrm{W}}=U$ and $J_{\mathrm{Re}}=J_{\mathrm{W}}=J$. Futhermore, we fix the exchange interaction by $J / U=0.2$ which leaves only one free parameter $U$.

Electron correlations are treated within the LA in which the correlated ground state $\left|\psi_{0}\right\rangle$ is obtained from the corresponding HF state $\left[\phi_{0}\right\rangle$ as follows

$$
\left|\psi_{0}\right\rangle=\exp \left[-\sum_{n} \eta_{n} O_{n}\right]\left|\phi_{0}\right\rangle
$$

The variational parameters $\eta_{n}$ are found by the minimization of the ground state energy $E_{\mathrm{LA}}=$ $\left\langle\psi_{0}|H| \psi_{0}\right\rangle /\left\langle\psi_{0} \mid \psi_{0}\right\rangle$. For the local operators $O_{n}$ we use $n_{m i \uparrow} n_{m i \downarrow}$ and $n_{m i} n_{m j}$ to reduce charge fluctuations within orbital $i$ and between two orbitals $i$ and $j$, respectively, while the operators $\mathbf{s}_{m i} \mathbf{s}_{m j}$ introduce spin-spin correlations. We calculate the ground state energy $E_{\Sigma A}$ by performing a variational second order expansion in powers of $\eta_{n}$. The respective quantities $\left\langle O_{n} H\right\rangle,\left\langle O_{n} H O_{n^{\prime}}\right\rangle$ and $\left\langle O_{n} O_{n^{\prime}}\right\rangle$ are evaluated by making use of the so-called $\mathbf{R}=0$ approximation in which only the leading local terms are preserved. This scheme allows to treat also stronger correlated systems and gives the correct atomic limit. More details may be found in reference [7].

We calculate the total energies of $R_{1}$ and of $R_{2}$ on $\mathrm{W}(110)$ for the respective geometries presented in figure 1. First, we solve the HF problem resulting from

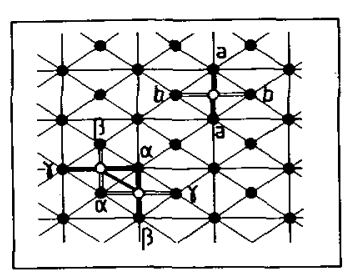

Fig. 1. - Schematic geometry of $\mathrm{Re}_{1}$ and $\mathrm{Re}_{2}$ (empty circles) on W(110) (full circles).

\footnotetext{
- Permanent address: Institute of Physics, Jagellonian University PL-30059 Kraków, Poland.
} 
(1) for different values of the magnetic moment $M_{m}$ (at $R_{1}$ ) and $M_{\mathrm{d}}$ (at one atom of $\mathrm{Re}_{2}$ ). Thereby, the LDOS at two neighbour $W$ atoms to the considered $\mathrm{Re}$, indicated in figure 1 by $\mathrm{a}, \mathrm{b}$ and $\alpha, \beta$, respectively, are determined selfconsistently. The HF energies are defined as follows

$$
\begin{aligned}
& E_{\mathrm{HF}, \mathrm{m}}=E_{\mathrm{HF}}(1)+2 E_{\mathrm{HF}}(\mathrm{a})+E_{\mathrm{HF}}(\mathrm{b})-3 E_{\mathrm{HF}}(\mathrm{W}),(3) \\
& E_{\mathrm{HF}, \mathrm{d}}=E_{\mathrm{HF}}(2)+E_{\mathrm{HF}}(\alpha)+E_{\mathrm{HF}}(\beta)-2 E_{\mathrm{HF}}(\mathrm{W}),(4)
\end{aligned}
$$

where $E_{\mathrm{HF}}(1)$ and $E_{\mathrm{HF}}(2)$ are the energies calculated for one $R e$ atom in $R_{1}$ and $R_{2}$ and $E_{H F}(W)$ is the reference energy of $W$ atom at $W(110)$. Thereby, we assume that $W_{b}$ and $W_{\gamma}$ atoms connected by a bond with a single Re atom are equivalent. Next, we determine the correlation energies $E_{\text {corr }, \mathrm{m}}$ and $E_{\text {corr,d }}$, defined in a similar way to equations $(3,4)$. Finally, the binding energy of $\mathrm{Re}_{2}$ is

$$
E_{0}=E_{\mathrm{HF}, \mathrm{m} 2}+E_{\mathrm{corr}, \mathrm{m}}-E_{\mathrm{HF}, \mathrm{d}}-E_{\mathrm{corr}, \mathrm{d}}-E_{\mathrm{rep}} \text {. }
$$

The repulsive energy involved in the formation of $\operatorname{Re}-$ Re bond is $E_{\text {rep }}=0.31 \mathrm{eV}$ [4].

\section{Results and discussion}

The main results of our study are summarized in figure 2. In the $\mathrm{HF}$ approximation $\mathrm{Re}_{2}$ becomes unstable at $U \approx 2.3 \mathrm{eV}$. Such a behaviour was already predicted earlier; at present we removed some inconsistency in the previous treatment of $E_{\mathrm{HF}, \mathrm{d}}$. After adding correlations, the value of $E_{0}$ is somewhat reduced in nonmagnetic states (for $U \leq 1.3 \mathrm{eV}$ ), but this reduction does not exceed $0.1 \mathrm{eV}$ and is far too small to destabilize $R_{2}$. In the region of magnetic $R_{1}$, the value of $E_{0}$ decreases faster and becomes negative at $U \approx$ $3.3 \mathrm{eV}$. We find there $M_{\mathrm{m}}>M_{\mathrm{d}}$, so one gains more magnetic energy for $\mathrm{Re}_{1}$ than for $\mathrm{Re}_{2}$. On the contrary, the gain from electron correlation is larger in less magnetic states of $\mathrm{Re}_{2}$. Thus, the currelation energy works against the dominating magnetic energy, i.e. stabilizes $\mathrm{Re}_{2}$.
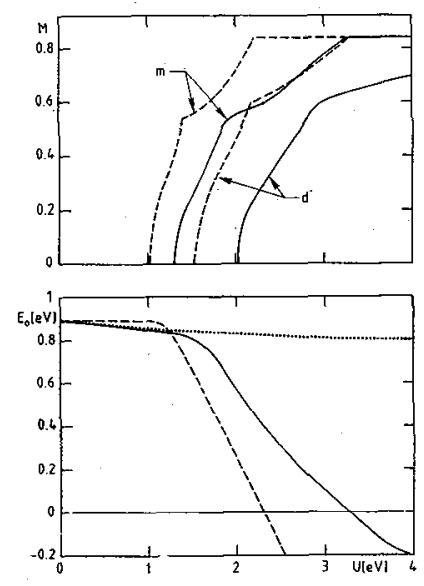

Fig. 2. - (a) Magnetic moments $M_{\mathrm{m}}$ and $M_{\mathrm{d}}$ and (b) bind$\mathrm{ng}$ energy $E_{0}$ of $\mathrm{Re}_{2}$ found in $\mathrm{HF}$ (dashed lines) and with ihe LA (full lines) for different values of $U$.
Several aspects of the presented analysis deserve attention. Since electron correlations suppress magnetic states (see [7] and the references therein), it is extremely important to include the saturation effects in the correlation energy [6]. The correlation energies presented in figure 2 are only about $40 \%$ of those obtained in SOP. Also, the Hund's-rule coupling $J$ is crucial for getting a magnetic ground state [7]. Indeed, by assuming $J=0$, one finds nonmagnetic solutions with stable $\mathrm{Re}_{2}$. Of course, there are still many effects which may influence our quantitative result. The most important correction to the calculated $E_{0}$ may come from a better treatment of the clusters formed by $\mathrm{Re}_{1}$ and $\mathrm{Re}_{2}$ and the surrounding $\mathrm{W}$ atoms. Further corrections are expected: (i) from the possible magnetic polarization of $\mathrm{W}$ atoms, (ii) from different local orientations of Re moments in $R_{2}$, (iii) due to the inaccuracy of $E_{\mathrm{rep}}$, (iv) if the value of $U_{\mathrm{W}}$ would be determined separately (but we find it encouraging that $U_{\mathrm{W}} \approx 3.4 \mathrm{eV}$ fits the values of the cohesive energy and bulk modulus) and ( $v$ ) from the spin-orbit coupling. Work on this line is in progress.

Summarizing, we have presented the simplest model for the $\mathrm{Re}_{2}$ instability on $\mathrm{W}(110)$, in which one may treat magnetism and electron correlations simultaneously. Our conclusion that the magnetism of $\mathrm{Re}_{1}$ is the principal reason for the $\operatorname{Re}_{2}$ instability is expected to be independent of all the details mentioned above. We hope that one should be able to understand also the observed behaviour of 5 d adatoms on W(110) [1-3] by extending the presented analysis. It would be very interesting to perform experiments to detect local moments of these adatoms at low coverage.

\section{Acknowledgment}

We thank Prof. J. Friedel for many stimulating discussions. The financial support of the Polish Research Project CPBP 01.03 is acknowledged.

[1] Tsong, T. T., Phys. Rev. B 6 (1972) 417.

[2] Bassett, D. W., Surf. Sci. 53 (1975) 74.

[3] Tsong, T. T. and Casanova, R., Phys. Rev. B 24 (1981) 3063;

Fink, H. W. and Ehrlich, G., Phys. Rev. Lett. 52 (1984) 1532.

[4] Bourdin, J. P., Desjonquères, M. C., Spanjaard, D. and Friedel, J., Surf. Sci. 157 (1985) L345.

[5] Bourdin, J. P., Desjonquères, M. C., Spanjaard, D. and Tréglia, G., Surf. Sci. 181 (1987) L183.

[6] Oleś, A. M. and Chao, K. A., Phys. Lett. 89A (1982) 420.

[7] Oleś, A. M. and Stollhoff, G., Phys. Rev. B 29 (1984) 314.

[8] Haydock, R., Heine, V. and Kelly, M. J., J. Phys. C 5 (1972) 2845. 